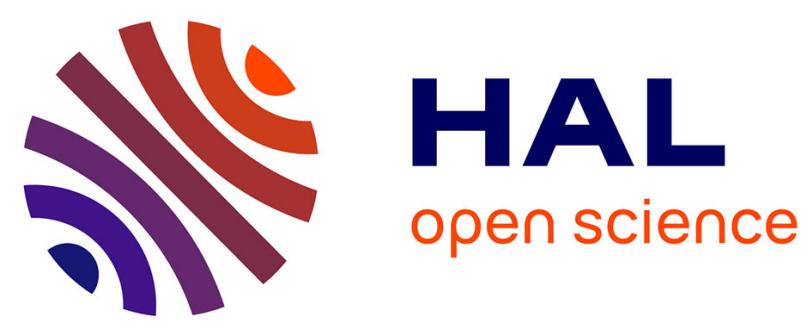

\title{
Development and validation of multitemporal image analysis methodologies for multirisk monitoring of critical structures and infrastructures
}

Sebastiano Serpico, Lorenzo Bruzzone, Giovanni Corsini, William J. Emery, Paolo Gamba, Andrea Garzelli, Grégoire Mercier, Josiane Zerubia, Nicola Acito, Bruno Aiazzi, et al.

\section{To cite this version:}

Sebastiano Serpico, Lorenzo Bruzzone, Giovanni Corsini, William J. Emery, Paolo Gamba, et al.. Development and validation of multitemporal image analysis methodologies for multirisk monitoring of critical structures and infrastructures. IEEE IGARSS - International Geoscience and Remote Sensing Symposium, Jul 2012, Munich, Germany. hal-00729089

\section{HAL Id: hal-00729089 \\ https://hal.inria.fr/hal-00729089}

Submitted on 7 Sep 2012

HAL is a multi-disciplinary open access archive for the deposit and dissemination of scientific research documents, whether they are published or not. The documents may come from teaching and research institutions in France or abroad, or from public or private research centers.
L'archive ouverte pluridisciplinaire HAL, est destinée au dépôt et à la diffusion de documents scientifiques de niveau recherche, publiés ou non, émanant des établissements d'enseignement et de recherche français ou étrangers, des laboratoires publics ou privés. 


\title{
DEVELOPMENT AND VALIDATION OF MULTITEMPORAL IMAGE ANALYSIS METHODOLOGIES FOR MULTIRISK MONITORING OF CRITICAL STRUCTURES AND INFRASTRUCTURES
}

\author{
Sebastiano B. Serpico ${ }^{1}$, Lorenzo Bruzzone ${ }^{2}$, Giovanni Corsini ${ }^{3}$, William J. Emery ${ }^{4}$, Paolo Gamba ${ }^{5}$, \\ Andrea Garzelli ${ }^{6}$, Gregoire Mercier ${ }^{7}$, Josiane Zerubia ${ }^{8}$, Nicola Acito ${ }^{3}$, Bruno Aiazzi ${ }^{6}$, Francesca \\ Bovolo $^{2}$, Fabio Dell'Acqua ${ }^{5}$, Michaela De Martino ${ }^{1}$, Marco Diani $^{3}$, Vladimir Krylov ${ }^{8}$, Gianni Lisini $^{5}$, \\ Carlo Marin', Gabriele Moser ${ }^{1}$, Aurelie Voisin ${ }^{8}$, Claudia Zoppetti ${ }^{6}$ \\ ${ }^{1}$ Univ. Genoa, via Opera Pia 11a, 16145 Genoa (Italy), e-mail: sebastiano.serpico@unige.it \\ ${ }^{2}$ Univ. Trento, Dept. of Information Eng. and Computer Science, via Sommarive 14, 38123 Povo (Italy) \\ ${ }^{3}$ Univ. Pisa, Dept. of Information Eng., via G. Caruso 16, 56122 Pisa (Italy) \\ ${ }^{4}$ Univ. Colorado Boulder, Aerospace Eng. Sciences, CO 80309-0431, Boulder (CO, USA) \\ ${ }^{5}$ Univ. Pavia, Dept. of Electronics, via Ferrata 1, 27100 Pavia (Italy) \\ ${ }^{6}$ Univ. Siena, via Roma 56, Siena, and IFAC-CNR, via Madonna del Piano 10, Sesto Fiorentino (Italy) \\ ${ }^{7}$ Telecom Bretagne, Technopole Brest-Iroise, CS 8381829238 Brest Cedex (France) \\ ${ }^{8}$ INRIA, Ayin team, 2004, Route des Lucioles, B.P.93, 06902 Sophia Antipolis (France)
}

\begin{abstract}
In the framework of the monitoring of structures and infrastructures from environmental disasters, the COSMOSkyMed constellation has a huge potential, thanks to up to metric spatial resolution, short revisit time, and the day/night all-weather acquisition capability ensured by SAR. This paper focuses on the scientific results of the project "Development and validation of multitemporal image analysis methodologies for multirisk monitoring of critical structures and infrastructures," funded by the Italian Space Agency. Several change-detection, data-fusion, and feature-extraction techniques, which were developed and experimentally validated in the project for COSMOSkyMed imagery and for their integration with other data sources (including very high resolution optical data), are described and examples of processing results are discussed.
\end{abstract}

Index Terms-Multirisk monitoring, urban areas, infrastructures, change detection, data fusion, feature extraction.

\section{INTRODUCTION}

The capability of monitoring structures and infrastructures from disasters such as fires, landslides, floods, etc., has a dramatic impact on human, social, and economic activities. The main contribution of remote sensing to this multirisk monitoring capability relies on the availability of very high resolution (VHR), all-weather, day/night, and short revisit time data. The COSMO-SkyMed (CSK $\left.{ }^{\circledR}\right)$ constellation has a huge potential, thanks to up to metric spatial resolution, revisit time as short as up to 12 hours, and the day/night and all-weather acquisition capability ensured by SAR.

This paper focuses on the scientific results of the project "Development and validation of multitemporal image analysis methodologies for multirisk monitoring of critical structures and infrastructures" (2010-2012; principal investigator: Prof. S. B. Serpico), funded by the Italian Space Agency (ASI) in the framework of the Announcement of Opportunity for CSK ${ }^{\circledR}$ data and devoted to development and experimental validation of image-analysis methods for multirisk monitoring of infrastructures and urban areas. The project has involved eight research units (see affiliations above) and addressed a number of challenging changedetection (CD), data-fusion, and feature-extraction problems. The next sections provide an overview of the proposed methodological approaches together with some examples of processing results obtained with CSK ${ }^{\circledR}$ images and other data sources (e.g., optical VHR data).

\section{CHANGE DETECTION FROM CSK® IMAGES AND POSSIBLE OTHER DATA SOURCES}

Several CD problems have been addressed, strongly focusing on the VHR capabilities of CSK ${ }^{\circledR}$ and encompassing pixelwise $\mathrm{CD}$ as well as detection of changes in small structures or buildings, and for port surveillance.

Pixelwise CD with VHR CSK® and multisensor data. A critical issue in pixelwise CD with VHR data is to characterize the spatial information associated with the image and correctly capture the geometrical structure that is usually apparent in VHR images of urban areas and infrastructures [2]. To this end, a novel method has been 
proposed that combines an edge-preserving Markov random field (MRF) model based on line processes, a parameterestimation strategy based on a dictionary of SAR-specific probability density functions (PDFs), and graph-cut techniques for Markovian energy minimization. The method is fully automatic and, thanks to the data-fusion capabilities of MRF models, is feasible for both single- and multichannel input images and has also been extended to multisensor data composed of both CSK ${ }^{\circledR}$ and optical VHR observations. Generalized Gaussians are used to model the PDFs of optical VHR data.

Experiments were conducted with $\mathrm{CSK}{ }^{\circledR}$ and QuickBird images acquired over Port-au-Prince (Haiti) before and after the 2010 dramatic earthquake. Qualitative validation through photo-interpretation and by comparison with ancillary data on the earthquake suggested the accuracy of the resulting change maps (Fig. 1; details can be found in [12]). These results are interpreted as due to the multisensor and VHR spatial modeling capability allowed by the proposed MRF and by the effectiveness of graph-cut algorithms for Markovian energy minimization.

Detection of changes over buildings. An approach has been developed based on two observations: i) in VHR SAR images, each building generates a clear double bounce; ii) a change in buildings implies a change in the double-bounce backscattering. The proposed method uses the Bayesian rule for compound classification to fuse the information about double-bounce lines, and about presence/absence of changes in the backscattering. Promising results have been obtained on a pair of spotlight VHR CSK $®$ images acquired over L'Aquila (Italy) before and after the earthquake occurred on April 6, 2009.

$C D$ for port surveillance. A method has been developed under the assumption that prior auxiliary information about the typical use of the areas is available (e.g., loading zones, storage tank areas, docking facilities). The method is based on two steps: 1) multiscale detection of changes in backscattering, and 2) detection of changes at the finest scale. Step 1 computes a pixel-based multilevel representation of the multitemporal information by applying the two-dimensional stationary wavelet transform to the logratio (LR) image [3], in order to roughly detect areas most probably associated with changes [4]. Step 2 is iterative and considers available prior information to define a proper set of multitemporal features that allow each specific change to be effectively characterized at the best resolution level. Which kind of feature and which CD technique should be involved in the process depend on the specific considered application. Experiments have been carried out on two spotlight CSK $®$ images, acquired on April 23 and 24, 2010, on the port of Livorno, Italy (see Fig. 4). Between the two acquisitions, many cars were moved in the cargo terminal area and, as an example, we could count the number of such moved cars (see Figs. 4.a and b, and Table I) and tankers were moved in the wet dock (see Figs. 4.c and d). These results were allowed by the multilevel information representation and by the proper characterization of car backscattering, modeled in terms of isolated scatterers [5] and formulated according to prior information.

Detection of small changes in VHR CSK® images. The problem has been approached by considering a general scheme based on a two-step procedure: first, the background characterization is accomplished by using multitemporal data; then, the detection of small changes is performed based on the results of the previous step. Generally, a threshold-based test has been adopted to discriminate the changed pixels between two images acquired at different times. Such a test is performed on a specific statistic that encompasses the relationships between the information content of the two images. The effectiveness of two CD schemes has been investigated. Given two intensity images $X$ and $Y$ acquired over the same area at different times, in order to detect the small changes occurred in $X$ with respect to $Y$, both considered $\mathrm{CD}$ schemes assume $Y$ as background and the pixels of $X$ that are anomalous with respect to the background are declared as changes. Anomalousness between each pixel in $X$ and the background is evaluated through a suitable similarity measure called test statistic. The first considered CD algorithm locally characterizes the background in terms of local mean and standard deviations, and uses the Mahalanobis distance as the test statistic. To mitigate the problem of the random fluctuation in the intensity value of the same pixel in the two different acquisitions, a linear prediction algorithm is entailed in the CD scheme.

In the second considered $\mathrm{CD}$ scheme for the detection of small changes, the test statistic is the conditional PDF evaluated for the intensity value of each pixel in $X$, conditioned to the value taken by $Y$ in the same spatial position. High values of this conditional PDF denote high degree of similarity between the pixels in the two images, whereas low values indicate that a change has occurred in $X$. The involved PDF-estimation problems have been addressed by making use of a nonparametric approach based on the kernel density estimator (KDE [6][7]) and by automatically optimizing its parameters by the plug-in approach (Silverman's rule [7]). KDE is very attractive for its capability of self adaptation to different and heterogeneous scenarios. To test the CD algorithms, two CSK ${ }^{\circledR}$ spotlight images acquired over Livorno have been analyzed. The experiments have been carried out on a parking area where the ground truth for small changes has been derived by visual inspection.

Land-cover mapping from VHR CSK® images. Due to the key role that land-cover thematic information plays in the use of $\mathrm{CD}$ products in risk-monitoring applications, the problem of land-cover mapping from VHR CSK ${ }^{\circledR}$ images has also been addressed by proposing novel techniques based on multiscale MRF and copula theory [1]. 


\section{FEATURE EXTRACTION FOR CHANGE DETECTION}

Novel procedures have also been developed in the project to combine enhanced feature extraction schemes with $\mathrm{CD}$ and to support the detection of changes even when only heterogeneous data are available. Different approaches have been considered, including statistical and geometrical features.

Improvement of $C D$ results based on geometrical features. A procedure has been designed to improve preexisting $\mathrm{CD}$ results by considering changes detected within geometrical features [8][9]. After the extraction of linear features from the scene, a "no-change" map corresponding to an area around matching features is extracted. This map may be used in conjunction with area-based or pixel-based change maps (such as those mentioned in Section 2) to refine their results. The method has been tested on pre- and post-event spotlight CSK ${ }^{\circledR}$ data over Port-au Prince (see examples of results in Fig. 2).

Information-theoretic feature extraction for $C D$. A second approach considered a spatial information-theoretic feature from multitemporal detected SAR images. The method is unsupervised and does not require accurate despeckling. Its steps are: local averaging; partitioning of the scatter plot of the two-date observations; mean-shift clustering; migration of the feature points toward cluster modes (the mode on the diagonal of the scatterplot for "no-change" pixels, those outside for "change" pixels); an appropriate scaling of the estimated joint distribution on the set $A$ of scatter points belonging to the most populated (no-change) cluster and on the set $B$ of scatter points belonging to the other clusters; and, finally, the computation of a multitemporal change feature by an information-theoretic formulation. Fig. 3 shows examples of results obtained with spotlight CSK ${ }^{\circledR}$ data of L'Aquila by a previous algorithm [10], by the proposed improved technique adopting mean-shift clustering, and by classical LR.

$C D$ from heterogeneous images. $\mathrm{CD}$ from a pair of heterogeneous images (e.g., different acquisition geometries or polarizations) has been addressed by evaluating the texture distance between local distributions extracted by colocated sliding windows at the two dates. The local distributions are characterized by non-parametric Edgeworth expansions while their comparison is expressed in terms of Kullback-Leibler divergence. Since local distributions may strongly differ in the case of heterogeneous data, a simulated joint distribution has been computed parametrically by copula theory [11] or non-parametrically by Kohonen's self organizing maps.

\section{CONCLUSION}

Several challenging image-analysis problems have been addressed in the project. Novel change detection, data fusion, and feature extraction techniques have been methodologically developed and experimentally validated with CSK $^{\circledR}$ and multisource data. The experiments suggested the effectiveness of the proposed methods in the application to diverse typologies (spotlight, stripmap, etc.) of CSK $®$ images of urban areas and infrastructures and their capability to take benefit from the information conveyed by CSK $\AA$, optical VHR imagery, and ancillary data.

\section{ACKNOWLEDGMENT}

This work was carried out in the framework of the project "Development and validation of multitemporal image analysis methodologies for multirisk monitoring of critical structures and infrastructures," funded by the Italian Space Agency (ASI). The support is gratefully acknowledged. Prof. Zerubia would also like to thank INRIA and the French Defense Agency (DGA) for partial financial support of a PhD student (A. Voisin) and a postdoc (V. Krylov) involved in this work.

\section{REFERENCES}

[1] A. Voisin, V. Krylov, G. Moser, S. B. Serpico, J. Zerubia. "Multichannel hierarchical image classification using multivariate copulas", IS\&T/SPIE Electronic Imaging 2012, Proc. of SPIE, volume 8296, 82960K, San Francisco (USA), January 22-26, 2012

[2] G. Moser, S. B. Serpico, "Edge-preserving classification of highresolution remote-sensing images by Markovian data fusion," Proc. of IGARSS-2009

[3] F. Bovolo, L. Bruzzone, "A detail-preserving scale-driven approach to change detection in multitemporal SAR images," IEEE Trans. Geosci. Rem. Sens., 43:2963- 2972, 2005

[4] R. J., Radke, S. Andra, O. Al-Kofahi, B. Roysam, "Image change detection algorithms: a systematic survey," IEEE Trans. Image Process., 14:294-307, 2005

[5] A. Lopes, E. Nezry, R. Touzi, H. Laur, "Structure detection and statistical adaptive speckle filtering in SAR images," Int. J. Remote Sens., 14:1735-1758, 1993

[6] H. Wolfgang, M. Müller, S. Sperlich, A. Werwatz, Nonparametric and Semiparametric Models, Springer-Verlag, 2004

[7] B.W. Silverman, Density Estimation for Statistics and Data Analysis, Chapman and Hall, 1986

[8] M. Negri, P. Gamba, G. Lisini, F. Tupin, "Junction-aware extraction and regularization of urban road networks in high resolution SAR images," IEEE Trans. Geosci. Remote Sensing, 44:2962-2971, 2006

[9] F. Dell'Acqua, P. Gamba, G. Lisini, "Change detection of multitemporal SAR data in urban areas combining feature-based and pixel-based techniques", IEEE Trans. Geosci. Remote Sensing, 44:2820-2827, 2006

[10] L. Alparone, B. Aiazzi, S. Baronti, A. Garzelli, F. Nencini, "Robust change analysis of SAR data through information-theoretic multitemporal features," Proc. of IGARSS-2007, pp. 3883-3886, July 23-28, 2007

[11] G. Mercier, G. Moser, S. B. Serpico, "Conditional copulas for change detection in heterogeneous remote sensing images," IEEE Trans. Geosci. Remote Sensing, 46:1428-1441, 2008

[12] L. Cianci, G. Moser, S. B. Serpico, "Change detection from very highresolution multisensor remote-sensing images by a markovian approach," Proc. of IEEE-GOLD-2012 (in press) 

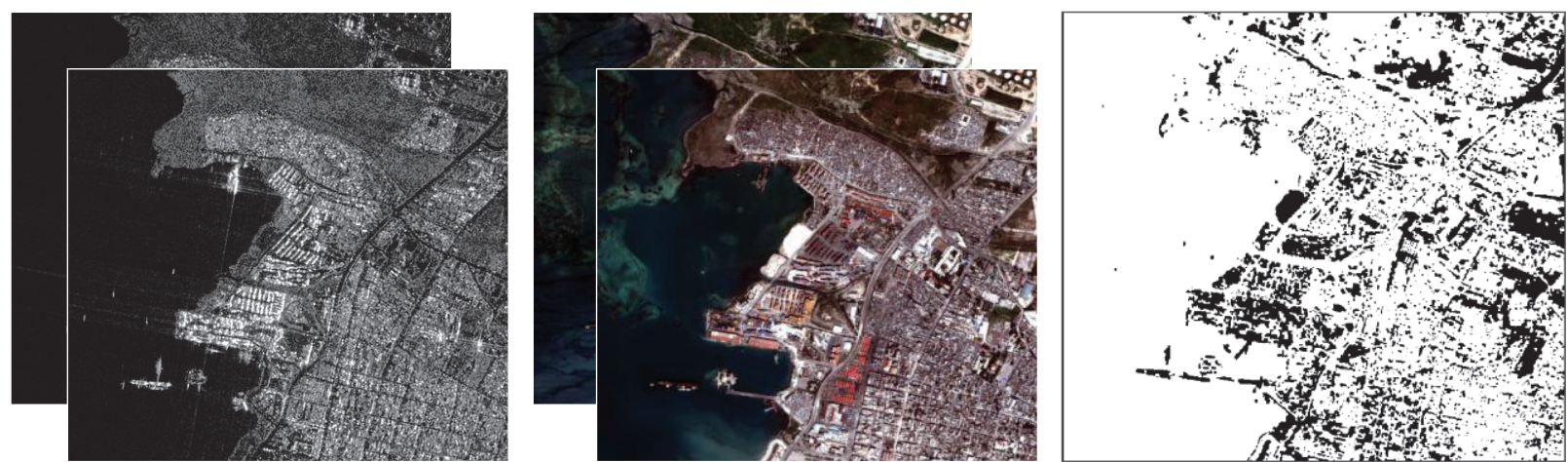

Fig. 1. CD results with multitemporal CSK ${ }^{\circledR}$ (left; COSMO-SkyMed Product - CASI - Agenzia Spaziale Italiana - 2009-2010) and QuickBird images (center; CDigitalGlobe, 2011) acquired over Port-au-Prince (Haiti) before and after the 2010 earthquake. Black and white in the change map (right) denote "change" and "no-change," respectively.
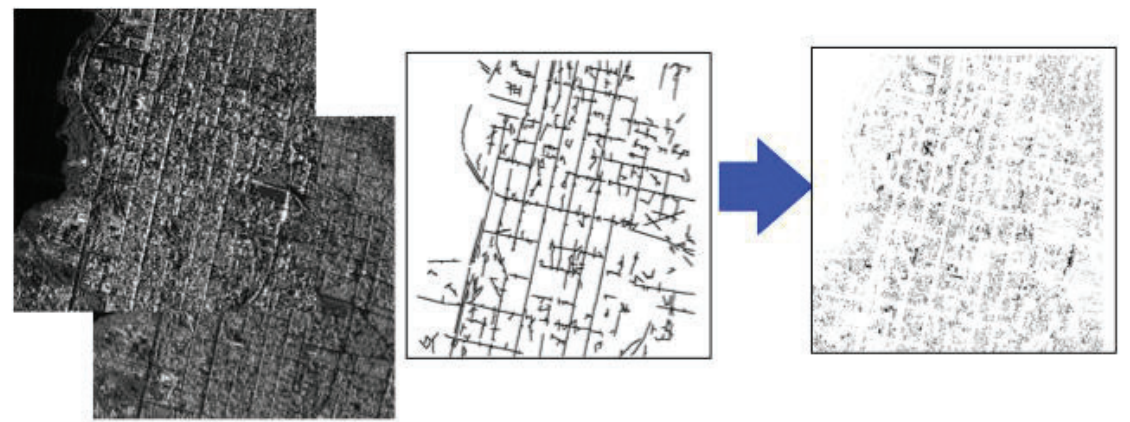

Fig. 2. Graphical representation of the work flow of the CD improvement procedure based on geometrical features: application to input CSK ${ }^{\circledR}$ images (COSMO-SkyMed Product -

CASI - Agenzia Spaziale Italiana - 20092010) of Port-au-Prince.
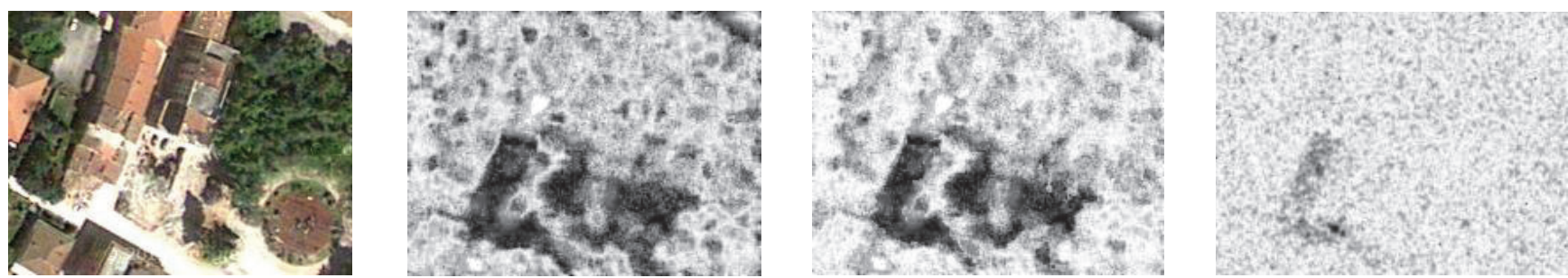

Fig. 3. Reference optical data of collapsed buildings over L'Aquila, via Campo di Fossa (left) and change features extracted from multitemporal CSK ${ }^{\circ}$ images of L'Aquila by (from left to right) the method in [10], the proposed technique, and LR.

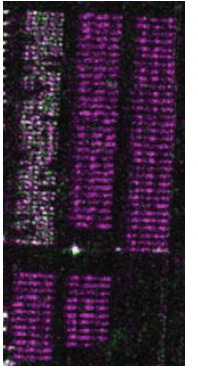

(a)

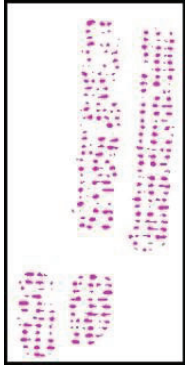

(b)

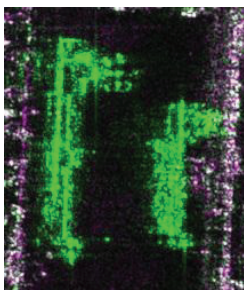

(c)

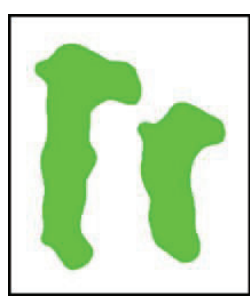

(d)
TAble 1 Specific Parameters ASSOCiated With Changed ARea Retrieved in Post-Processing

\begin{tabular}{c|c|c}
\hline Parameter & Retrieved & Reference \\
\hline $\begin{array}{c}\text { Length of cargo } \\
\text { ships }\end{array}$ & Ship 1:117m & Ship 1:116m \\
\cline { 2 - 3 } & Ship 2: $85 \mathrm{~m}$ & Ship 2: $74 \mathrm{~m}$ \\
\hline $\begin{array}{c}\text { \# of cars in cargo } \\
\text { terminal }\end{array}$ & 284 & 249 \\
\hline
\end{tabular}
$\square$ Changes due to a backscattering decrease; $\square$ Changes

Fig. 4 RGB multitemporal composition (R:04/24/2010, G:04/23/2010, B:04/24/2010) and CD map of two full-scene sub-sets (COSMOSkyMed Product - CASI - Agenzia Spaziale Italiana -2010) showing (a-b) cargo terminal where new cars were stocked and (c-d) a wet dock zone where two cargo ships were anchored. 